\title{
QUALIDADE DA ÁGUA DE UMA PISCICULTURA EM TANQUES- REDE NO RIO SÃO JOSÉ DOS DOURADOS, ILHA SOLTEIRA - SÃO PAULO
}

Juliana Heloisa Pinê Américo ${ }^{1}$

\section{Vanderlei Previato ${ }^{2}$}

\section{Sérgio Luís de Carvalho ${ }^{3}$}

RESUMO: O aporte excessivo de nutrientes provenientes de resíduos de ração e metabólitos de peixes nos sistemas de produção aquícola tem provocado alterações nos parâmetros de qualidade das águas. $O$ objetivo deste trabalho foi avaliar a qualidade da água de uma piscicultura em tanques-rede localizada no Rio São José dos Dourados, município de Ilha Solteira - São Paulo, através das análises de nitrogênio total, fósforo total, demanda bioquímica de oxigênio (DBO) e oxigênio dissolvido na água. As análises dos parâmetros de nitrogênio total, fósforo total, DBO e oxigênio dissolvido foram realizadas no Laboratório de Saneamento da FEIS-UNESP com base nos Métodos para Análises de Águas Potáveis e Residuárias Standard Methods for Examination of Water and Wastewater - APHA-AWWA-WPCF 1998. Todos os parâmetros de qualidade de água avaliados enquadraram- se no padrão estabelecido pela legislação para águas doces destinadas à aquicultura e à atividade de pesca. De acordo com dados gerados neste estudo, conclui-se que a piscicultura com sistema de cultivo em tanques-rede Proaqua instalada no Rio São José dos Dourados, Ilha Solteira - SP não está alterando a qualidade da água no que diz respeito aos parâmetros de nitrogênio total, fósforo total, DBO e oxigênio dissolvido, No entanto, deve ser realizado um monitoramento constante da qualidade da água para que a mesma não seja degradada e a piscicultura na região não se torne uma atividade inviável economicamente e ambientalmente.

Palavras-chave: Nitrogênio. Fósforo. Tilápia.

\footnotetext{
${ }^{1}$ Doutoranda em Aquicultura, Centro de Aquicultura da UNESP. E-mail: americo.ju@gmail.com

${ }^{2}$ Doutorando em Ciência dos Materiais, Faculdade de Engenharia de llha Solteira (UNESP). E-mail: vpreviato@ig.com.br

${ }^{3}$ Prof. Adjunto, Faculdade de Engenharia de Ilha Solteira (UNESP). E-mail: sergicar@bio.feis.unesp.br
} 


\section{INTRODUÇÃO}

O cultivo de peixes em tanques-rede é uma modalidade de sistema intensivo de produção que surge como uma alternativa para o aproveitamento dos corpos hídricos inexplorados pela piscicultura convencional. Este sistema de produção possui uma contínua renovação de água visando à manutenção da qualidade da água dentro das estruturas de criação, através da remoção de dejetos e metabólitos produzidos pelos organismos cultivados (COLT e MONTGOMERY,1991).

No entanto, o intenso desenvolvimento da aquicultura em tanques-rede em longo prazo pode provocar alterações na qualidade da água, principalmente no que se refere à introdução de nutrientes (fósforo e nitrogênio) e matéria orgânica nestes ambientes. De acordo com Beveridge (2004) e Pillay (2004), 30\% da matéria orgânica presente na ração utilizada na produção do pescado em sistemas de cultivo não são aproveitadas e permanecem disponíveis no ambiente aquático.

Segundo Baccarin e Camargo (2005), este aporte de matéria orgânica nos ecossistemas aquáticos propicia o processo de eutrofização artificial que pode afetar os sistemas de produção de organismos aquáticos devido à redução da concentração de oxigênio dissolvido e aumento da concentração de nitrogênio e fósforo na água, que pode causar blooms de algas e bactérias.

Alguns gêneros de algas que se desenvolvem em ambientes eutrofizados podem causar toxidez aos organismos aquáticos e assim degradarem os potenciais produtos da aquicultura, como por exemplo, o "gosto de barro" nos filés de peixe conhecido como "off flavor", causado pela exposição dos animais à geosmina, um subproduto do metabolismo de algas fixadoras de nitrogênio (BARAK et al., 2003).

Assim, os sistemas de produção utilizados pela aquicultura, como a produção de peixes em tanques-rede em grandes reservatórios, devem ser manejados através de um constante monitoramento da qualidade da água, não apenas dentro e entre os tanquesrede, mas também no ambiente que envolve essa atividade para que haja uma garantia da qualidade do produto bem como da sustentabilidade dos sistemas intensivos de produção de peixes (MARENGONI, 2006).

O objetivo deste trabalho foi avaliar a qualidade da água de uma piscicultura em tanques-rede localizada no Rio São José dos Dourados, município de llha Solteira - São 
Paulo, através das análises de nitrogênio total, fósforo total, demanda bioquímica de oxigênio (DBO) e oxigênio dissolvido na água.

\section{MATERIAL E MÉTODOS}

\subsection{Caracterização da área de estudo e pontos de amostragem}

A área de estudo localiza-se na piscicultura Proaqua no Rio São José dos Dourados, município de llha Solteira na região Noroeste do Estado de São Paulo. A piscicultura é constituída por 90 tanques-rede com cultivo de tilápia do Nilo (Oreochromis niloticus) e apresenta uma área cultivada de 4 hectares.

Quatro pontos de amostragem foram definidos na área de estudo e devidamente georreferenciados, estes denominados P1 (ponto central do sistema de cultivo em tanques-rede); P2 (entrada de água da piscicultura); P3 (canal do rio fora da área da piscicultura) e P4 (água de lançamento da piscicultura). Na Tabela 1 encontram-se as coordenadas geográficas dos pontos amostrais localizados na piscicultura Proaqua no Rio São José dos Dourados, município de llha Solteira - SP.

Tabela 1. Coordenadas geográficas dos pontos de amostragem da piscicultura Proaqua localizada no Rio São José dos Dourados, município de llha Solteira - SP

\begin{tabular}{cc}
\hline Pontos Amostrais & Coordenadas geográficas \\
\hline P1 & $20^{\circ} 26^{\prime} 28^{\prime \prime} \mathrm{S}$ \\
& $51^{\circ} 14^{\prime} 53^{\prime \prime} \mathrm{W}$ \\
P2 & $20^{\circ} 26^{\prime} 34^{\prime \prime} \mathrm{S}$ \\
& $51^{\circ} 14^{\prime} 51^{\prime \prime} \mathrm{W}$ \\
& $20^{\circ} 26^{\prime} 40^{\prime \prime} \mathrm{S}$ \\
& $51^{\circ} 14^{\prime} 59^{\prime \prime} \mathrm{W}$ \\
& $20^{\circ} 26^{\prime} 37^{\prime \prime} \mathrm{S}$ \\
& $51^{\circ} 15^{\prime} 01^{\prime \prime} \mathrm{W}$ \\
\hline
\end{tabular}


As amostras de água dos quatro pontos da área de estudo foram coletadas mensalmente, a $80 \mathrm{~cm}$ da superfície, no período de janeiro a agosto de 2008 abrangendo as estações seca e chuvosa do ano.

\subsection{Análises dos parâmetros de qualidade de água}

As análises dos parâmetros de nitrogênio total, fósforo total, DBO e oxigênio dissolvido foram realizadas no Laboratório de Saneamento da FEIS-UNESP com base nos Métodos para Análises de Águas Potáveis e Residuárias - Standard Methods for Examination of Water and Wastewater (APHA-AWWA-WPCF, 1998). Na Tabela 2 encontram-se os métodos e equipamentos utilizados para as análises dos parâmetros selecionados para avaliação da qualidade da água da piscicultura em estudo.

Tabela 2. Métodos e equipamentos utilizados para análise dos parâmetros selecionados para avaliação da qualidade da água da piscicultura Proaqua no Rio São José dos Dourados, Ilha Solteira - SP

\begin{tabular}{|c|c|c|}
\hline Parâmetros & Método & Equipamentos \\
\hline \multirow{4}{*}{ Nitrogênio total } & & COD Reator/Hatch \\
\hline & Digestão por ácido persulfato & Autoclave Vertical Phoenix \\
\hline & e espectrofotométrico & Espectrofotômetro \\
\hline & & Odyssey/Hatch/DR-2500 \\
\hline \multirow{4}{*}{ Fósforo total } & & COD Reator/Hatch \\
\hline & Digestão por ácido persulfato & Misturador Biomixer QL-901 \\
\hline & e espectrofotométrico & Espectrofotômetro \\
\hline & & Odyssey/Hatch/Dr-2500 \\
\hline \multirow{3}{*}{ DBO } & & Titulador \\
\hline & Método de Winkler modificado & Estufa para DBO Tecnal TE \\
\hline & & 390 \\
\hline Oxigênio dissolvido & Método de Winkler modificado & Titulador \\
\hline
\end{tabular}

\section{RESULTADOS E DISCUSSÃO}


De acordo com a Resolução Conama 357/2005 para águas doces de classe 2 (águas destinadas à aquicultura e à atividade de pesca), quando o nitrogênio for fator limitante para eutrofização, nas condições estabelecidas pelo órgão ambiental competente (valor de nitrogênio total após oxidação), sua concentração não deverá ultrapassar 2,18 mg. $\mathrm{L}^{-1}$ para ambientes lóticos.

Em relação à concentração deste nutriente, todos os valores registrados nos quatro pontos amostrais da piscicultura Proaqua no Rio São José dos Dourados ficaram abaixo do padrão estabelecido na legislação, com exceção do ponto central dos tanques-rede na amostra de água do mês de abril de 2008. Este pico de nitrogênio no mês de abril ocorreu porque a coleta foi realizada logo após o fornecimento de ração para os peixes o que contribui para o acúmulo deste nutriente na água, ressaltando-se que não só os restos de ração, mas também a excreção dos peixes influenciam na introdução de nutrientes e matéria orgânica nos ambientes aquáticos.

As menores concentrações de nitrogênio total foram encontradas no canal do rio que se encontra fora da área da piscicultura (P3), evidenciando a influência desta atividade nos demais pontos (Tabela 3 ).

Tabela 3. Concentração de nitrogênio total na água, em $m g \cdot L^{-1}$, nos pontos $P 1$ : ponto central do sistema de tanques-rede, P2: entrada de água da piscicultura, P3: canal do rio fora da piscicultura e P4: água de lançamento da piscicultura Proaqua no Rio São José dos Dourados, Ilha Solteira $\mathrm{SP}$, no período de janeiro à agosto de 2008

\begin{tabular}{ccccccccc}
\hline Pontos & \multicolumn{7}{c}{ Meses } \\
\cline { 2 - 8 } Amostrais & Jan & Fev & Mar & Abr & Mai & Jun & Jul & Ago \\
\hline P1 & 0,6 & 0,4 & 0,6 & 33,8 & 0,5 & 0,8 & 0,7 & 0,9 \\
P2 & 0,7 & 0,6 & 0,4 & 0,7 & 0,7 & 0,5 & 0,6 & 0,6 \\
P3 & 0,1 & 0,1 & 0,2 & 0,3 & 0,2 & 0,3 & 0,2 & 0,2 \\
P4 & 0,2 & 0,3 & 0,3 & 0,8 & 0,5 & 0,4 & 0,3 & 0,3 \\
\hline
\end{tabular}

As concentrações de fósforo total nos quatro pontos de amostragem não ultrapassaram o padrão estabelecido para ambientes lóticos $\left(0,1 \mathrm{mg} \cdot \mathrm{L}^{-1}\right)$ segundo a Resolução Conama 357/2005. No ponto central dos tanques-rede (P1) registraram-se principalmente no período chuvoso concentrações de fósforo total maiores em relação 
aos demais pontos amostrais o que demonstra a influência da piscicultura no aporte deste nutriente na água (Tabela 4).

Tabela 4. Concentração de fósforo total na água, em $\mathrm{mg} \cdot \mathrm{L}^{-1}$, nos pontos $\mathrm{P} 1$ : ponto central do sistema de tanques-rede, P2: entrada de água da piscicultura, P3: canal do rio fora da piscicultura e P4: água de lançamento da piscicultura Proaqua no Rio São José dos Dourados, Ilha Solteira SP, no período de janeiro a agosto de 2008.

\begin{tabular}{ccccccccc}
\hline Pontos & \multicolumn{7}{c}{ Meses } \\
\cline { 2 - 8 } Amostrais & Jan & Fev & Mar & Abr & Mai & Jun & Jul & Ago \\
\hline P1 & 0,02 & 0,02 & 0,02 & 0,05 & 0,03 & 0,02 & 0,03 & 0,03 \\
P2 & 0,02 & 0,01 & 0,01 & 0,02 & 0,04 & 0,03 & 0,02 & 0,02 \\
P3 & 0,01 & 0,01 & 0,01 & 0,02 & 0,02 & 0,02 & 0,01 & 0,01 \\
P4 & 0,02 & 0,01 & 0,01 & 0,02 & 0,03 & 0,02 & 0,02 & 0,02 \\
\hline
\end{tabular}

A demanda bioquímica de oxigênio (DBO) expressa a presença de matéria orgânica na água, constituindo-se em importante indicador de qualidade das águas naturais. Indica a magnitude do consumo de oxigênio $\left(\mathrm{mg}^{\mathrm{L}} \mathrm{L}^{-1}\right.$ ) pelas bactérias na decomposição da matéria orgânica. A presença de alto teor de matéria orgânica pode conduzir à completa extinção do oxigênio na água, provocando o desaparecimento de toda vida aquática.

A Resolução Conama 357/2005 limita o valor de DBO em até $5 \mathrm{mg} \cdot \mathrm{L}^{-1}$ de $\mathrm{O}_{2}$, valor este superior ao registrado em todos os pontos de amostragem da piscicultura durante 0 período de monitoramento (Tabela 5). Como durante o monitoramento os valores de DBO não ultrapassaram $1,48 \mathrm{mg} \cdot \mathrm{L}^{-1}$, provavelmente os resíduos orgânicos da piscicultura foram assimilados sem comprometer a concentração de oxigênio dissolvido na água.

Tabela 5. Demanda bioquímica de oxigênio, em mg. $\mathrm{L}^{-1}$, nos pontos $\mathrm{P} 1$ : ponto central do sistema de tanques-rede, P2: entrada de água da piscicultura, P3: canal do rio fora da piscicultura e P4: água de lançamento da piscicultura Proaqua no Rio São José dos Dourados, Ilha Solteira - SP, no período de janeiro a agosto de 2008.

\begin{tabular}{ccccccccc}
\hline Pontos & \multicolumn{7}{c}{ Meses } \\
\cline { 2 - 9 } Amostrais & Jan & Fev & Mar & Abr & Mai & Jun & Jul & Ago \\
\hline P1 & 1,04 & 0,84 & 1,25 & 1,31 & 1,25 & 1,08 & 1,48 & 1,14 \\
\hline
\end{tabular}




$\begin{array}{lllllllll}\text { P2 } & 0,87 & 0,77 & 1,62 & 1,25 & 1,29 & 0,91 & 0,87 & 0,99 \\ \text { P3 } & 0,38 & 0,33 & 0,41 & 0,52 & 0,82 & 0,83 & 0,44 & 0,61 \\ \text { P4 } & 0,57 & 0,67 & 1,24 & 1,17 & 1,08 & 0,89 & 0,91 & 1,01\end{array}$

Em relação à concentração de oxigênio dissolvido, observou-se que os valores encontrados em todos os pontos da piscicultura durante o monitoramento enquadram-se no padrão estabelecido pela Resolução Conama 357/2005 para corpos d'água classe 2, onde a concentração deste gás dissolvido na água em qualquer amostra não deve ser inferior à $5 \mathrm{mg} \cdot \mathrm{L}^{-1}$. Para piscicultura, segundo Kubitza (2000) a concentração mínima de oxigênio dissolvido que deve ser mantida é de $4 \mathrm{mg}^{-\mathrm{L}^{-1}}$, quando esta concentração chega a valores abaixo de $2 \mathrm{mg} \cdot \mathrm{L}^{-1}$ pode ocorrer excessivo estresse e mortalidade de peixes.

Quando a concentração de oxigênio dissolvido atinge 45 a 50\% de saturação (aproximadamente 3 a $3,5 \mathrm{mg} / \mathrm{L}$ a $28-30^{\circ} \mathrm{C}$ ), a tilápia começa a reduzir sua atividade e, portanto, o consumo de oxigênio (ROSS e ROSS, 1983). Observou-se no presente estudo que em todos os meses as maiores concentrações de oxigênio dissolvido na água foram registradas no canal do rio fora da piscicultura (P3), o que evidência a influencia da piscicultura neste parâmetro (Tabela 6).

Tabela 6. Concentração de oxigênio dissolvido na água, em mg. $\mathrm{L}^{-1}$, nos pontos P1: ponto central do sistema de tanques-rede, P2: entrada de água da piscicultura, P3: canal do rio fora da piscicultura e P4: água de lançamento da piscicultura Proaqua no Rio São José dos Dourados, Ilha Solteira - SP, no período de janeiro a agosto de 2008.

\begin{tabular}{ccccccccc}
\hline Pontos & \multicolumn{7}{c}{ Meses } \\
\cline { 2 - 8 } Amostrais & Jan & Fev & Mar & Abr & Mai & Jun & Jul & Ago \\
\hline P1 & 5,98 & 5,86 & 6,91 & 7,13 & 7,71 & 7,65 & 7,66 & 8,07 \\
P2 & 5.76 & 5,66 & 6,89 & 7,06 & 7,75 & 7,58 & 8,01 & 8,12 \\
P3 & 6,74 & 6,54 & 7,10 & 7,30 & 8,06 & 8,18 & 8,29 & 8,45 \\
P4 & 5,68 & 5,48 & 6,83 & 7,14 & 7,74 & 7,73 & 8,05 & 8,10 \\
\hline
\end{tabular}

\section{CONCLUSÃO}

De acordo com dados gerados neste estudo, conclui-se que a piscicultura com sistema de cultivo em tanques-rede Proaqua instalada no Rio São José dos Dourados, 
Ilha Solteira - SP não está alterando a qualidade da água no que diz respeito aos parâmetros de nitrogênio total, fósforo total, DBO e oxigênio dissolvido, pois todos os parâmetros de qualidade de água avaliados enquadraram- se no padrão estabelecido pela legislação para águas doces destinadas à aquicultura e à atividade de pesca, fato que pode ser atribuído à pequena área cultivada em relação á vazão do rio.

No entanto, deve ser realizado um monitoramento constante da qualidade da água para que a mesma não seja degradada e a piscicultura na região não se torne uma atividade inviável economicamente e ambientalmente.

\section{REFERÊNCIAS}

APHA - AMERICAN PUBLIC HEALTH ASSOCIATION. - AWWA. AMERICAN WATER WORKS ASSOCIATION - WATER ENVIRONMENTAL FEDERATION - WPCF Standard methods for the examination of water and wastewater. $20^{\text {th }}$. Washington: APHA, 1998.

BACCARIN, A. E.; CAMARGO, A. F. M. Characterization and evaluation of the impact of feed management on the effluents of Nile Tilapia (Oreochromis niloticus). Culture

Brazilian Archives of Biology and Technology, Curitiba, v. 48, n.1, p. 81-90, 2005.

BARAK, Y.; CYTRYN, E.; GELFAND, I.; KROM, M.; van RIJN, J. Phosphorus removal in a marine prototype, recirculating aquaculture system. Aquaculture, Amsterdam v.220, p.313-326, 2003.

BEVERIDGE, M.C.M. 2004. Cage aquaculture. 3th. Oxford, UK: Blackwell Publishing Ltd. 2004. $376 \mathrm{p}$.

COLT, J.; MONTGOMERY J. M. Aquaculture production systems. Journal of Animal Science, EUA, v. 69, p. 4183-4192, 1991.

CONSELHO NACIONAL DO MEIO AMBIENTE - CONAMA. Resolucao n`357, de $\mathbf{1 7}$ de março de 2005. Disponivel em: <http:www.mma.gov.br/port/conama> Acesso: 15 jun. 2013.

KUBITZA, F. 2000. Tilápia: tecnologia e planejamento na produção comercial. Jundiaí: $F$. KUBITZA, 2000. 289p. 
MARENGONI, N.G. Produção de tilápia do Nilo Oreochromis niloticus (linhagem chitralada), cultivada em tanques-rede, sob diferentes densidades de estocagem. Archivos de Zootecnia, Córdoba, v.55, n.210, p.127-138, 2006.

PILLAY, T.V.R. 2004. Aquaculture and the environment. 2th. Oxford: Blackwell Publishing, 2004. 94p.

ROSS, B.; ROSS, L. G. The oxigen requeriments of Oreochromis niloticus under adverse conditions. In: FISHELSON, L.; YARON, Z. (eds.) International symposium on tilapia in aquaculture.Tel Aviv University, Israel 1983. p.134-143. 